\title{
Síntese e caracterização de Microesferas (PMMA) Superparamagnéticas via polimerização em suspensão
}

\section{Synthesis and characterization of Supeparamagnetics Microspheres (pmma) via suspension polymerization}

\author{
Paulo Emilio Feuser ${ }^{1}$; Marcio Nele de Souza ${ }^{2}$
}

\section{Resumo}

Nanopartículas magnéticas (NPMs) tem encontrado diversas aplicações nas áreas tecnológicas e biomédicas. O objetivo deste trabalho é a preparação e caracterização de microesferas de PMMA contendo NPMs revestidas com ácido oleico (NPMs-AO). Para a preparação das NPMs-AO utilizou-se método de co-precipitação em meio aquoso. Para a preparação das microesferas superparamagnéticas utilizou-se a técnica de polimerização em suspensão. As microesferas apresentaram uma distribuição de tamanho de partículas de aproximadamente 150um e uma morfologia esférica. A partir das analises de cromatografia de permeação em gel (GPC) determinou-se o peso molecular médio (Mw) das microesferas magnéticase verificou-se uma variação do Mw dependendo da concentração de NPMs-AO na reação. Para analise das propriedades magnéticas utilizou-se o magnetômetro de amostra vibrante (MAV). As microesferas apresentaram propriedades superparamagnéticas e um valor de magnetização de saturação (Ms) de aproximadamente $8 \mathrm{emu} / \mathrm{g}$ de NPMs. Portanto pode conclui se que é possível obter microesferas superparamagnética para uma determinada aplicação, seja ela, biomédica ou tecnológica. Palavras-chave: Magnetita. Microesferas. Polímeros magnéticos.

\begin{abstract}
Magnetics nanoparticles (NPMs) has found many applications in biomedical and technological areas. The objective of this work is the preparation and characterization of PMMA microspheres containing NPMs coated with oleic acid (NPMs-AO). For the preparation of MNPs-AO was used the coprecipitation method in an aqueous medium. For the preparation of the superparamagnetic microspheres used in suspension polymerization technique. The microspheres showed a size distribution particles of approximately $150 \mathrm{um}$ and a spherical morphology. From the analysis of gel permeation chromatography (GPC) determined the number average molecular weight (Mw) of the magnetics microspheres and there was a variation in the $\mathrm{Mw}$ depending on the concentration of MNPs-AO in this reaction. To analyze the magnetic properties used the vibrating sample magnetometer (MAV). The microspheres showed superparamagnetic properties and a value of saturation magnetization (Ms) of about $8 \mathrm{emu} / \mathrm{g}$ MNPs. Therefore you can conclude that it is possible to obtain superparamagnetics microspheres for a particular application, either, biomedical or technological.
\end{abstract}

Keywords: Magnetite. Microspheres. Magnetics polymers.

${ }^{1}$ Mestre em Engenharia Química, Universidade Federal de Santa Catarina; paulofeuser@hotmail.com.

2 Docente da Escola de Química da Universidade Federal do Rio de Janeiro - EQA - UFRJ; nele@eq.ufrj.br. 


\section{Introdução}

Microesferas magnéticas têm sido amplamente utilizados para fins analíticos e clínicos como, por exemplo, na imobilização de enzimas, sequenciamentos de ácido nucléico, purificação de proteínas, isolamento de células, monitoramento celular e na hipertermia (BETTENCOURT; ALMEIDA, 2012; CHUNG; LEE, 2008; TOMITAKA; YAMADA; TAKEMURA et al., 2012).

A aplicação biomédica é um novo campo para as microesferas magnéticas, desde que estas apresentem tamanho uniforme, biocompatibilidade e propriedades superparamagnéticas (XU et al., 2006; FAN et al., 2009). Uma das áreas mais promissoras na utilização das nanopartículas magnéticas (NPMs) é a vetorização de fármacos anticancerígenos através de sistemas de liberação de fármacos almejando uma distribuição mais seletiva e assim um aumento do índice terapêutico (FAN et al., 2009; KAKAR et al., 2013).

Os parâmetros mais importantes para a aplicação das NPMs é o controle do tamanho das partículas, aspecto morfológico e as propriedade magnética do material final. Assim um controle eficiente durante sua preparação é de fundamental importância (LIU et al., 2004). O material magnético mais utilizado é a Magnetita $\left(\mathrm{Fe}_{3} \mathrm{O}_{4}\right)$, por apresentar biocompatibilidade e uma saturação de magnetização superior a outros óxidos de ferro (ROSENSWEIG, 1985). A encapsulação das NPMs tem como finalidade aumentar sua estabilidade, prevenindo assim aglomerações influenciando diretamente no comportamento magnético das microesferas (LANDFESTER; RAMIRES, 2003).

Particularmente o uso do poli (metacrilato de metila) (PMMA) se destaca nessa área, uma vez que esse material é biocompatível e o monômero metacrilato de metila (MMA) apresenta baixa toxicidade. Por essa razão, o MMA e o PMMA são amplamente empregados para preparação de cimentos dentais, cimentos ósseos, próteses ósseas e em medicamentos (BETTENCOURT; ALMEIDA, 2012). O material polimérico proporciona estabilidade físico-química e também biocompatibilidade dependendo da área de atuação (LU et al, 2007). Dependendo do capo de aplicação das microesferas magnéticas o material final deve apresentar tamanho e distribuição uniforme, não apresentar sedimentação, ser atóxico e não ter vazamento férreo,.(ZHANG; KOHLER; ZHANG, 2002).

O objetivo deste trabalho é a preparação e a caracterização das microesferas com propriedades supeparamagnéticas via polimerização em suspensão.

\section{Materiais e Métodos}

Para a síntese das microesferas magnéticas foram utilizados os seguintes reagentes: metacrilato de metila (MMA), peróxido de benzoila (BPO) e poliálcool vinilico (PVA), todos provenientes da VETEC química e água destilada. Para a preparação das NPMs revestidas com ácido oleico (NPMsAO) utilizou-se o cloreto férrico $\left(\mathrm{ClFe}_{3} \cdot 5 \mathrm{H}_{2} \mathrm{O}\right)$, sulfato ferroso $\left(\mathrm{FeSO}_{4} \cdot 7 \mathrm{H}_{2} \mathrm{O}\right)$, hidróxido de amônia $\left(\mathrm{NH}_{4} \mathrm{OH}\right)$, ácido oléico $\left(\mathrm{C}_{18} \mathrm{H}_{34} \mathrm{O}_{2}\right)$ e acetona todos provenientes da VETEC Química e água destilada $\left(\mathrm{H}_{2} \mathrm{O}\right)$.

As NPMs-AO foram preparadas pelo método de co-precipitação em meio aquoso. com a adição de $\mathrm{FeSO}_{4} \cdot 7 \mathrm{H}_{2} \mathrm{O}$ e $\mathrm{ClFe}_{3} \cdot 6 \mathrm{H}_{2} \mathrm{O}$ na proporção molar $1: 1$ e logo em seguida adicionou-se $\mathrm{NH}_{4} \mathrm{OH}$ controlando o pH inicial ( $\mathrm{pH}$ 1) e final ( $\mathrm{pH} 10)$ da reação, utilizando temperatura ambiente e uma velocidade de agitação de $700 \mathrm{rpm}$ por $30 \mathrm{~min}$. Na segunda etapa adicionou-se o ácido oleico (AO) para estabilizar as NPMs, mantendo a agitação em 700rpm por mais $30 \mathrm{~min}$. Depois de estabilizadas o material foi lavado com acetona para retirar o excesso de AO e logo em seguida a amostra é centrifugada e lavada várias vezes com acetona, até que o excesso de $\mathrm{AO}$ seja removido totalmente. 
As microesferas de PMMA contendo NPMs foram preparadas via polimerização em suspensão. $\mathrm{Na}$ primeira etapa em um reator de vidro sob uma agitação vigorosa (600rpm) a temperatura ambiente adicionou-se $200 \mathrm{ml}$ de agua destilada com $0,5 \%$ de PVA. Quando a temperatura atingiu $80^{\circ} \mathrm{C}$ o MMA, o BPO e as NPMs-AO (5 ou 10\% em relação ao MMA) foram adicionados ao reator. Após 4 hrs de reação as microesferas magnéticas foram lavadas com água destilada e filtradas. Em seguida as amostras foram secas em uma estufa a vácuo $\left(60^{\circ} \mathrm{C}\right)$ por $24 \mathrm{hrs}$. $\mathrm{Na}$ tabela 1 pode se observar as variáveis utilizadas para preparação das microesferas magnéticas.

Tabela 1 - Preparação das microesferas superparamagnéticas

\begin{tabular}{lcccc}
\hline Amostras & $\begin{array}{c}\text { NPMs-AO } \\
\text { (g) }\end{array}$ & $\begin{array}{c}\text { Tempo de } \\
\text { reação } \\
\text { (hrs) }\end{array}$ & T $^{\circ} \mathrm{C}$ & $\begin{array}{c}\text { Agitação } \\
\text { (1pm) }\end{array}$ \\
F1 & $\mathrm{X}$ & 4 & 80 & 800 \\
F2-NPMs & 2 & 4 & 80 & 800 \\
F3-NPMs & 4 & 4 & 80 & 800 \\
\hline
\end{tabular}

Fonte: os autores

Para a caracterização da $\mathrm{Fe}_{3} \mathrm{O}_{4}$ revestidas com ácido oleico utilizou-se a técnica de microscopia eletrônica de transmissão (MET) e para identificação do material magnético utilizou-se um difratômetro de raio-X (DRX) da marca Shimadzu XRD 6000. Para a caracterização das microesferas magnéticas utilizouse a microscopia óptica (um estéreo microscópio binocular Nikon SMZ800) para determinar a morfologia. A distribuição de tamanho e o índice de polidispersão (IP) das foi analisada em um analisador de tamanho de partículas, Malversizer 2000 (Malvern Instruments). As medições foram feitas em triplicata $(n=3)$. A massa molar foi determinada pela técnica de cromatografia de permeação em gel (GPC) (cromatógrafo Waters 600E, equipado com três colunas Ultrastyragel e detector refratométrico Waters 2414) e as propriedades magnéticas foram analisadas por magnetômetro de amostra vibrante (MAV).

\section{Resultados e Discussão}

Utilizando a técnica de microscopia eletrônica de transmissão (TEM) pode-se obter com clareza o tamanho e a morfologia das NPMs-AO. A Figura 1 mostra as NPMs-AO com um tamanho médio de aproximadamente $10 \mathrm{~nm}$. Quando o material magnético é sintetizado, é essencial que este apresente um tamanho de partícula na faixa de 5-18 $\mathrm{nm}$, pois é nesta faixa de tamanho que o material apresenta propriedades superparamagnéticas (GNANAPRAKASH et al., 2002). A adição de AO além de tornar mais hidrofóbica a superfície das NPMs previne a aglomeração, aumentando a estabilidade e mantendo um alto grau de saturação da magnetização.

\section{Figura 1 - TEM das NPMs- AO}

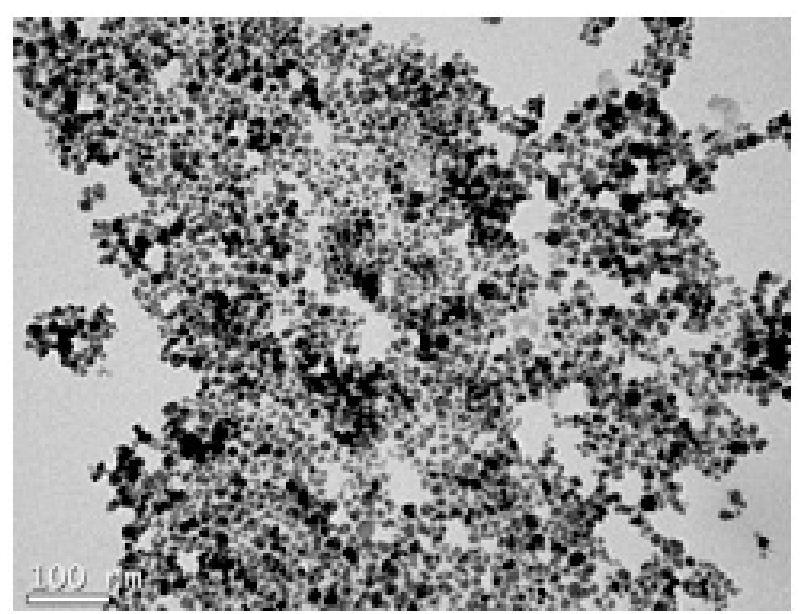

Fonte: os autores

A identificação das NPMs-AO pode ser feita utilizando a técnica de DRX. A partir da Figura 2 pode-se ver a formação de uma estrutura cristalina, característica da NPMs. As características dos picos $(220,311,400,422,511$ e 440) são característicos da magnetita $\left(\mathrm{Fe}_{3} \mathrm{O}_{4}\right)$ (GNANAPRAKASH et al., 2002; HONG et al., 2008). O diâmetro médio estimado dos cristalitos foi de $13 \mathrm{~nm}$, calculado a partir da largura a meia altura do pico de difração (311), através da equação de Scherrer, Eq. (1). 


$$
\tau=\frac{\kappa \lambda}{\beta \cos \theta}
$$

Figura 2 - DRX das NPMs-AO

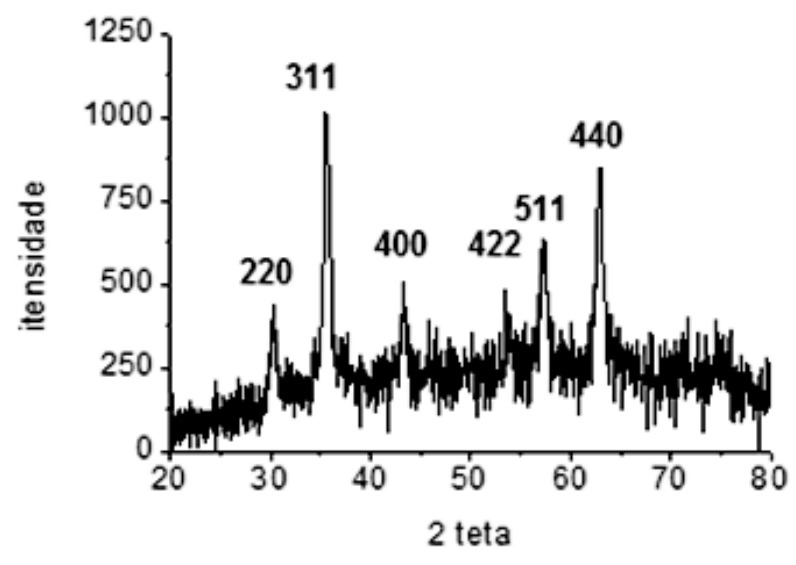

Fonte: os autores

A partir das analises de microscopia óptica (Figura 3) pode se verificar que as microesferas magnéticas preparadas via polimerização em suspensão apresentaram uma morfologia esférica.

Figura 3 - Micrografia do microesferas superparamagnéticas: F2-NPMS (A) e F3-NPMS (B).

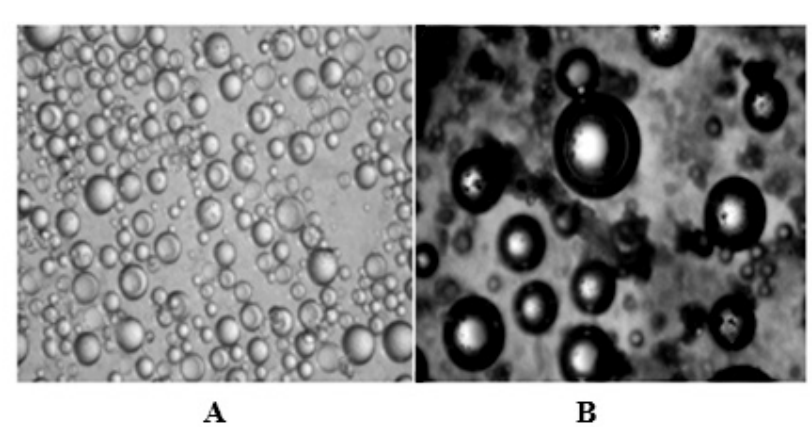

Fonte: os autores

A DTP das microesferas magnéticas foi de 154 \pm 1 ,6um, $159 \pm 2$,1 um e $151 \pm 2$,4um com IP de 0,48 $\pm, 0,40,48 \pm 0,4$ e $0,49 \pm 0,3$ para as formulações $F 1$,
F2-NPMs e F3-NPMs respectivamente. A DTP das formulaçõesnãoapresentaram diferença significativa e a quantidade de NPMs-AO utilizada no meio reacional não interfere na DTP. O alto IP revela uma DTP bimodal. No entanto o peso molecular médio (Mw) das microesferas diminui significativamente quando aumentamos a concentração de NPMs-O. Desta forma quanto maior o teor de NPMs-OA menor é a razão monômero/iniciador, sendo assim, são formadas cadeias de maior massa molar. Este aumento na $\mathrm{Mw}$ pode ser devido à reação do iniciador com o ferro presente no meio reacional, formando e reduzindo a concentração de radicais livres (HONG et al., 2008). Outra possibilidade é a reação dos radicais livres com o AO que recobre as NPMs, gerando radicais com baixa reatividade e por consequência resultando na obtenção de cadeias de maior massa molar.

Tabela 2 - Peso molecular médio (Mw) das microesferas superparamagnéticas

\begin{tabular}{cc} 
Amostras & $\begin{array}{c}\text { Massa Molar } \\
\text { Média (Mw - Da) }\end{array}$ \\
F1 & 340.000 \\
F2-NPMs & 280.000 \\
F3-NPMs & 240.000 \\
\hline
\end{tabular}

Fonte: os autores

A Figura 4 mostra a analise de DRX das microesferas magnéticas (F3-NPMs) com os picos característicos das NPMs-AO. Pode se dizer que o método de encapsulamento das NPMs-AO via polimerização em suspensão foi satisfatório, pois não houve qualquer alteração na estrutura cristalina do material magnético. (HONG et al., 2008). O pico mais amplo na faixa de $20^{\circ}$ a $30^{\circ}$ é atribuído à característica do polímero amorfo, neste caso o PMMA. (FAN et al., 2009). 
Figura 4 - DRX das microesferas superparamagnéticas.

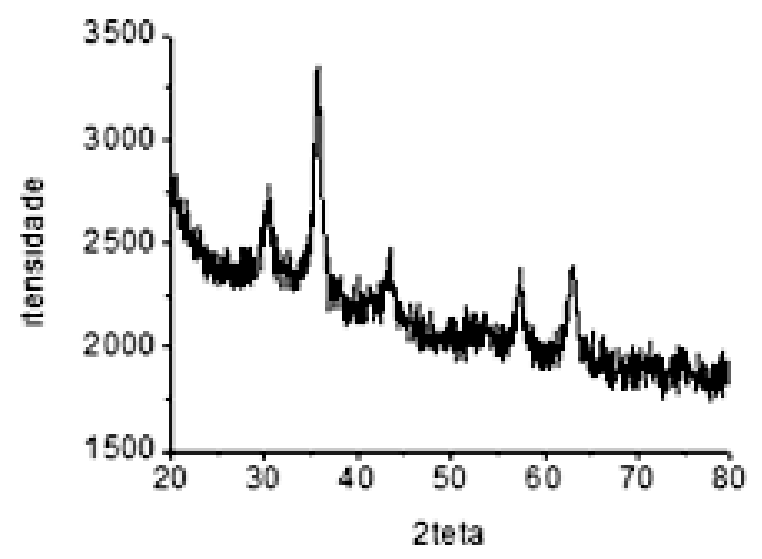

Fonte: os autores

$\mathrm{O}$ valor da magnetização de saturação $(\mathrm{Ms})$ foi de $61 \mathrm{emu} / \mathrm{g}$ e $8 \mathrm{emu} / \mathrm{g}$ para as NPMs-AO e F3NPMs respectivamente (Figura $5 \mathrm{a}$ e $5 \mathrm{~b}$ ). Segundo Hong et al. (2008) e Landfester e Ramires (2003) o baixo valor de Ms das microesferas pode ser atribuída a oxidação das NPMs durante a reação de polimerização.

A partir das Figuras $5 \mathrm{c}$ e $5 \mathrm{~d}$ pode se verificar que as NPMs-AO e as microesferas apresentaram comportamento superparamagnético, ou seja, sem a presença da curva de histereses, quando expostos há temperatura ambiente (ZHAO; SAATCHI; HAFELI, 2009).
Figura 5 - Propriedades magnéticas campo magnético de 20KOe: NPMs-AO (A) e F3-NPMs (B) e em maior escala 4KOe: NPMs-AO (C) e F3NPMs (D).

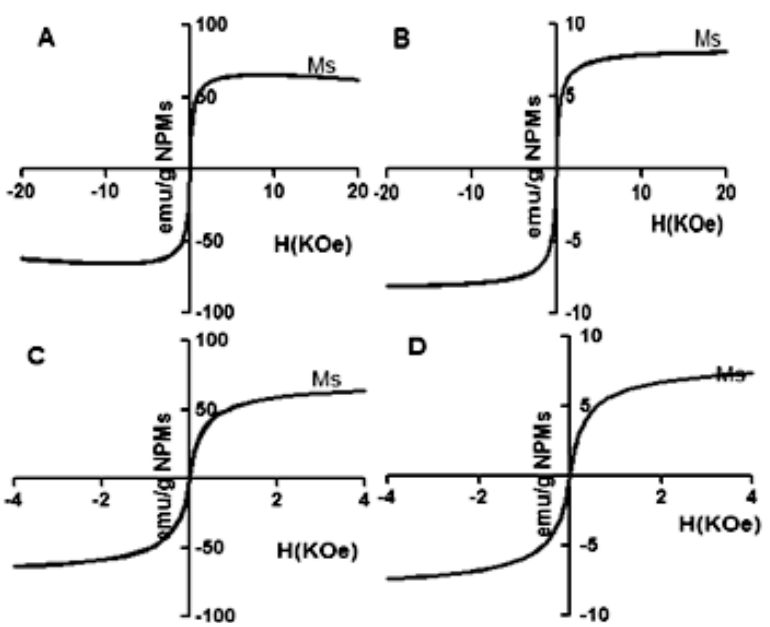

Fonte: os autores

\section{Conclusão}

O método de co-precipitação em meio aquoso para preparação de nanopartículas magnéticas majoritariamente compostas de magnetita se mostrou eficiente. A técnica de polimerização em suspensão também se mostrou eficiente para o encapsulamento das nanopartículas magnéticas revestidas com ácido oleico. Com tudo este trabalho mostra que é possível obter microesferas superparamagnéticas com diferentes massas molares, e dependendo do campo de aplicação este pode ser fator determinante.

\section{Agradecimentos}

Este trabalho teve o apoio financeiro do CNPQ.

\section{Referências}

BETTENCOURT, A.; ALMEIDA, A. J. Poly(methyl methacrylate) particulate carriers in drug delivery. Journal of Microencapsulation, London,,v. 29, n. 4, p. 353-367, 2012. 
CHUNG, T. H.; LEE, W. C. Preparation of styrenebased, magnetic polymer microspheres by a swelling and penetration process. Reactive and Functional Polymers, Amsterdam, v. 68, n. 10, p. 1441-1447, 2008.

FAN, L-H.; LUO, Y.-L.; CHEN, Y-S.; ZHANG, C-H.; WEI, Q.-B. Preparation and characterization of $\mathrm{Fe} 3 \mathrm{O} 4$ magnetic composite microspheres covered by a P(MAH-co-MAA) copolymer. Journal of Nanoparticle Research, Dordrecht, v. 11, n. 2, p. 449-458, 2009.

GNANAPRAKASH， G.; MAHADEVAN， S.; JAYAKUMAR, T.; KALYANASUNDARAM, P.; PHILIP, J.; RAJ, B. Effect of initial $\mathrm{pH}$ and temperature of iron salt solutions on formation of magnetite nanoparticles. Materials Chemistry and Physics, v.103, p. 168-175, 2002.

HONG, R. Y.; FENG, B.; CAI, X.; LIU, G.; LI, H. Z.; DING, J.; ZHENG, Y.; WEI, D. Z. Doubleminiemulsion preparation of $\mathrm{Fe} 3 \mathrm{O} 4 /$ poly(methyl methacrylate) magnetic latex. Journal of Applied Polymer Science, New York, v. 112, n. 1, p. 89-98, 2008.

KAKAR, S.; BATRA, D.; SINGH, R.; NAUTIYAL, U. Magnetic microspheres as magical novel drug delivery system: A review. Journal of Acute Disease, Hainan, v. 2, n. 1, p. 1-12, Jan. 2013.

LANDFESTER, K; RAMIRES, L. P. Encapsulated magnetite particles for biomedical application. Journal of Physics, v.15, p. S1345-S1361, 2003.

JOURNAL LIU, X.; MA, Z.; XING, J.; LIU, H. Preparation and characterization of amino-silane modified superparamagnetic silica nanospheres. Journal of Magnetism and Magnetic Materials, Amsterdam, v. 270, n. 1/2, p. 1-6, 2004.

LU, M.; BAI, S.; YANG, K.; SUN, Y. Synthesis and characterization of magnetic polymer microspheres with a core-shell structure. China Particuology, v.5, p. 180-185, 2007.
ROSENSWEIG, E. R. Ferrohydrodynamics. New York: Dover Publications, 1985.

TOMITAKA, A.; YAMADA, T.; TAKEMURA, Y. Magnetic nanoparticle hyperthermia using pluronic-coated $\mathrm{Fe}_{3} \mathrm{O}_{4}$ nanoparticles: an in vitro study. Journal of Nanomaterials, New York, p. 1-5, 2012.

XU, X.; DENG, C.; GAO, M.; YU, W.; YANG, P.; ZHANG, P. Synthesis of Magnetic Microspheres with Immobilized Metal Ions for Enrichment and Direct Determination of Phosphopeptides by Matrix-Assisted Laser Desorption Ionization Mass Spectrometry. Advanced Materials, v.18, p. 3289 3293, 2006.

ZHANG, Y.; KOHLER, N.; ZHANG, M. Surface modification of superparamagnetic magnetite nanoparticles and their intracellular uptake. Biomaterials, Surrey, v. 23, n. 7, p. 1553-1561, 2002.

ZHAO, H.; SAATCHI, K.; HAFELI, U. O. Preparation of biodegradable magnetic microspheres with poly(lactic acid)-coated magnetite. Journal of Magnetism and Magnetic Materials, Amsterdam, v. 321, n. 10, p. 1356-1363, 2009.

Recebido em 27 Junho 2013-Received on June 27, 2013. Aceito em 19 Setembro, 2013 - Accepted on September 19, 2013. 